\title{
AVALIAÇÃO SOROLÓGICA DE Parainfluenzavirus Tipo 1, Salmonella spp., Mycoplasma spp. E Toxoplasma gondii EM AVES SILVESTRES
}

\author{
Guilherme Augusto Marietto GonÇALVES ${ }^{1}$, Silvia Maria DE AlmeidA ${ }^{2}$, LuCILENE GranuZZI \\ CAMOSSI $^{3}$, HÉLIO LANGONI ${ }^{4}$, RAPHAEL LUCIO ANDREATTI FILHO ${ }^{4}$ \\ ${ }^{1}$ Médico Veterinário, Doutor, Universidade Estadual Paulista Júlio de Mesquita Filho, Faculdade de Medicina \\ Veterinária e Zootecnia de Botucatu, Botucatu, SP, Brasil.gmarietto_ornito@fmvz.unesp.br \\ ${ }^{2}$ Médica Veterinária autônoma, Botucatu, SP, Brasil. \\ ${ }^{3}$ Universidade Estadual Paulista Júlio de Mesquita Filho, Faculdade de Medicina Veterinária e Zootecnia de \\ Botucatu, Botucatu, SP, Brasil. \\ ${ }^{4}$ Professores Doutores da Universidade Estadual Paulista Júlio de Mesquita Filho, Faculdade de Medicina \\ Veterinária e Zootecnia de Botucatu, Botucatu, SP, Brasil.
}

\begin{abstract}
A doença de Newcastle, salmonelose e micoplasmose são enfermidades importantes para a avicultura e a toxoplasmose é uma zoonose comum no ambiente urbano. No presente estudo, pesquisaram-se evidências sorológicas dessas enfermidades em aves cativas e de vida livre com a aplicação das técnicas de soroaglutinação rápida em placa, inibição da hemaglutinação e aglutinação direta modificada. Em 117 amostras de soro sanguíneo, 20 apresentaram anticorpos para algumas das enfermidades
\end{abstract}

avaliadas, sendo constatada a presença de anticorpos para Toxoplasma gondii, Mycoplasma gallisepticum e Salmonella spp. A espécie Amazona aestiva foi a que apresentou o maior número de indivíduos soropositivos no estudo (13/20). Além disso, verificamos a primeira detecção de anticorpos para $T$. gondii em aves de rapina das espécies Mivalgo chimachima e Rupornis magnirostris.

PALAVRAS-CHAVE: Doença de Newcastle; micoplasmose; saúde aviária; sorologia; toxoplamose.

\section{SEROLOGICAL EVALUATION OF Parainfluenzavirus Type 1, Salmonella spp., Mycoplasma spp., And Toxoplasma gondii IN WILD BIRDS}

\section{ABSTRACT}

Newcastle disease, salmonellosis and mycoplamosis are the most important infectious diseases in poultry. Toxoplamosis is a common disease in urban environment. The present study investigated serologic evidence of these diseases in captive and wildlife birds, with rapid plate agglutination test, haemagglutination inhibition test, and modified agglutination test. In a total of 117 blood serum samples, 20 showed the presence of Toxoplasma gondii, Mycoplasma gallisepticum, and Salmonella spp. antibodies. Amazona aestiva was the specie with the highest number of positive individuals (13/20). We also verified the first detection of $T$. gondii antibodies in birds of prey from Mivalgo chimachima and Rupornis magnirostris species.

KEYWORDS: Avian health; mycoplasmosis; Newcastle disease; serology; toxoplamosis. 


\section{INTRODUÇÃO}

O exame sorológico é um método laboratorial que visa ao estudo da presença e/ou mensuração das reações antígeno-anticorpo por meio da presença de imunoglobulinas no soro sanguíneo após a exposição do hospedeiro a um determinado agente estranho, ou seja, mede uma resposta específica do organismo frente a um antígeno. $\mathrm{O}$ objetivo de sua realização é a identificação de lotes positivos para infecções propondo medidas de controle e erradicação.

A doença de Newcastle, também conhecida como pseudo-peste-aviária, é causada por um vírus do gênero Avulavirus (Parainfluenzavirus), pertencente à família Paramyxoviridae, sendo o Parainfluenzavirus tipo 1 (APMV-1) o mais importante (CATOLLI et al., 2011). É uma doença de grande impacto econômico na avicultura mundial, fazendo parte da lista de notificação obrigatória da Organização Mundial de Saúde Animal (OIE). A transmissão se dá principalmente por aerossóis, podendo ser disseminada por longas distâncias (LOMINICZI et al., 1998; CATOLLI et al., 2011).

Toxoplasma gondii é um coccídeo da família Sarcocystidae causador da toxoplasmose (ELMORE et al., 2010). É um parasita intestinal de felídeos e um grande número de animais domésticos e selvagens foram descritos como hospedeiros intermediários (inclusive humanos). A infecção já foi registrada em aproximadamente 200 espécies de mamíferos, contaminados principalmente pela ingestão de bradizóitos de $T$. gondii (SILVA et al., 2006). Em aves, a infecção sintomática é incomum, sendo normalmente inaparente ou latente (DUBEY, 2002), porém é uma doença zoonótica muito importante para o ser humano.

Salmonella enterica subespécie enterica é uma bactéria da família Enterobacteriaceae que, em aves, pode causar três doenças distintas: pulorose (sorotipo Pullorum), tifo aviário (sorotipo Gallinarum) e paratifo aviário (causado pelos demais sorotipos que não sejam os anteriores). De aproximadamente 2500 sorotipos conhecidos de $S$. enterica subs. enterica, cerca de 90 são comuns em casos de infecções em humanos e em animais, incluindo as aves, podendo ou não causar sintomatologia de paratifo aviário (TINDALL et al., 2005; LUTFUL KABIR, 2010).

A micoplasmose é uma enfermidade causada por bactérias do gênero Mycoplasma pertencentes à classe Mollicutes, que causam os quadros de doença respiratória crônica $(M$, gallisepticum) e sinovite infecciosa (M. synoviae), sendo $M$. gallisepticum a espécie de maior importância econômica. Pode ser transmitida por via horizontal, através de fômites, aerossóis, ração e água contaminada, ou vertical devido à proximidade do ovário com os sacos aéreos (LEVISOHN \& KLEVEN, 2000).

A monitoria de doença de Newcastle, Salmonella e Mycoplasma é uma necessidade da indústria avícola brasileira, sendo requerida e regulamentada no Brasil por meio do Programa Nacional de Sanidade Avícola (MAPA, 2009), que inclui também a influenza aviária. A toxoplasmose não faz parte das doenças monitoradas pelos órgãos brasileiros de defesa sanitária em aves, mas, pelo seu potencial zoonótico, a participação das aves no ciclo epidemiológico dessa enfermidade merece um pouco mais de atenção pelos órgãos de defesa sanitária animal e humana.

O presente trabalho avaliou a presença de imunoglobulinas referentes à Parainfluenzavirus APMV 1, T. gondii, Salmonella spp., Mycoplasma gallisepticum e $M$. synoviae em aves silvestres com o objetivo de identificar a circulação dos referentes agentes etiológicos.

\section{MATERIAL E MÉTODOS}

Foram estudadas 117 aves, durante os anos de 2008-2009, pertencentes à fauna brasileira em processo de reabilitação para a vida-livre alojadas no Projeto Centroflora e também atendidas no Laboratório de Ornitopatologia da Faculdade de Medicina Veterinária e Zootecnia da Universidade Estadual Paulista, ambos localizados no município de Botucatu, estado de São Paulo (Brasil),

Para a realização dos testes, utilizaram-se amostras de soro sanguíneo. As coletas foram realizadas entre 8:00 e 10:00 horas, por meio de contenção física, respeitando-se um limite de 15 minutos entre contenção/coleta/liberação das aves e, nesse período, dedicou-se no máximo 5 minutos para coleta do sangue para cada ave, dentro dos viveiros onde as mesmas estavam alojadas.

$\mathrm{O}$ sangue foi coletado por punção da veia jugular com agulha hipodérmica $0,45 \times 13-26 \mathrm{G}^{1 / 2}$ estéril e seringa de $1,0 \mathrm{~mL}$ estéril, sendo que a quantidade de sangue variou conforme a espécie e o porte de cada ave $(0,4$ a $1,0 \mathrm{~mL})$. Após a coleta, o sangue foi condicionado em microtubos de polipropileno de $1,5 \mathrm{~mL}$ e, após a formação de 
coágulo (em temperatura ambiente), foi mantido e encaminhado sob refrigeração a $5^{\circ} \mathrm{C}$ até $\mathrm{o}$ processamento laboratorial.

Após a chegada ao laboratório, o material foi processado imediatamente, sendo todos os testes realizados no mesmo dia da coleta de sangue. Inicialmente, o soro foi separado do coágulo por centrifugação a $5.000 \mathrm{rpm}$ por cinco minutos em temperatura controlada de $5^{\circ} \mathrm{C}$.

A pesquisa para Salmonella foi realizada por meio do método de soroaglutinação rápida em placa (SAR). Para Mycoplasma, utilizou-se o SAR, confirmando-se os resultados com a técnica de Inibição da Hemaglutinação - método beta $\left(\mathrm{HI}_{\beta}\right)$, conforme SANTOS (2009). Para parainfluenzavirus APMV 1, utilizou-se o $\mathrm{HI}_{\beta}$ e para T. Gondii, aplicou-se a técnica de aglutinação direta modificada (MAT), com antígeno fixado pela formalina, conforme técnica descrita por DESMONTS \& REMINGTON (1980).

Para a detecção de anticorpos IH específicos contra o PMV-1, utilizou-se o teste de inibição da hemaglutinação (IH). $\mathrm{O}$ vírus usado no teste de IH constitui-se de estirpe La Sota derivada de vacina comercial passada em ovos embrionados. Os soros monoespecíficos utilizados como controles positivo e negativo foram preparados em aves SPF (galinhas livres de patógenos específicos). Foram utilizadas microplacas de poliestireno, contendo 96 cavidades, com fundo em "V". O ensaio foi desenhado de acordo com o método beta, no qual diluições seriadas do soro são misturadas com concentrações fixas e constantes de vírus. Os soros foram diluídos com um volume padrão de PBS $(0,05 \mathrm{~mL})$ variando entre $1: 2$ a 1:2048. A cada diluição, foram adicionados $0,05 \mathrm{~mL}$ de antígeno contendo quatro unidades hemaglutinantes (UHA). Os níveis de anticorpos foram expressos como sendo a recíproca da mais alta diluição na qual a hemaglutinação foi inibida em 100\%.

No exame para anticorpos contra $T$. gondii, as amostras de soro foram diluídas em microplacas de fundo chato, com adição de $150 \mu \mathrm{L}$ de PBS $0,01 \mathrm{M} \mathrm{pH} 7,2 \mathrm{em}$ todas as cavidades utilizadas. $\mathrm{Na}$ primeira cavidade foram adicionados $10 \mu \mathrm{L}$ de soro (diluição 1:16) e, após homogeneização, transferiram-se $50 \mu \mathrm{L}$ para outra cavidade, equivalendo a diluição $1: 64$, procedendo-se assim até diluição 1:1024. A seguir, $25 \mu \mathrm{L}$ de cada diluição do soro foram transferidos para as respectivas cavidades de microplacas com fundo em "V" e $25 \mu \mathrm{L}$ de 2-mercaptoetanol 0,2 M diluídos em PBS 0,01 M pH 7,2 e $50 \mu \mathrm{L}$ do antígeno diluídos em solução tampão borato $\mathrm{pH}$ 8,7 foram adicionados às cavidades. As placas foram seladas com filme plástico, homogeneizadas por um minuto e incubadas à temperatura ambiente por 12 horas, procedendo-se à leitura da reação. O teste foi considerado positivo quando houve formação de uma película cobrindo pelo menos metade do fundo da cavidade e negativo quando houve a formação de "botão de fundo".

Para a triagem das amostras de sorospositivos para Salmonella, utilizou-se o método de soroaglutinação rápida em placa (SAR), sendo a confirmação do teste realizada por meio de detecção do genoma pela técnica de reação em cadeia da polimerase (Polymerase Chain Reaction - PCR). No teste de SAR, utilizou-se um antígeno colorido disponível no comércio. Para isso, $50 \mu \mathrm{L}$ de soro foi misturado com $50 \mu \mathrm{L}$ de antígeno, sendo homogeneizado com bastão de vidro em placa de leitura para SAR. As amostras que apresentaram formação de grumos uniformes e homogêneos durante o período de dois minutos foram consideradas positivas. Para cada teste realizado, utilizaram-se soros controle positivo, produzido em aves SPF, e negativo, obtido de aves (Gallus gallus domesticus), comprovadamente livres de salmonelas.

Para Mycoplasma gallisepticum e $M$. Synoviae, utilizou-se a SAR, confirmando-se os resultados com a técnica de Inibição da Hemaglutinação - método beta. O teste consistiu em adicionar partes iguais de antígeno e soro, homogeneizando-se a mistura e, após dois minutos, verificou-se a presença de grumos, indicando a formação da reação de antígenoanticorpo. Para confirmação dos resultados positivos no SAR, aplicou-se a técnica de $\mathrm{HI}_{\beta}$, utilizando-se amostras vacinais vivas Cevac ${ }^{\circledR}$ MGF (Ceva, Paulínia, Brasil), aplicando-se a mesma metodologia e critérios de avaliação da técnica empregada para APMV 1.

\section{RESULTADOS}

Foram avaliadas 117 aves, de 13 espécies diferentes, pertencentes a cinco ordens distintas (Tabela 1): Papagaio verdadeiro (Amazona aestiva), Papagaio do mangue (A. amazonica), Arara canindé (Ara ararauna), Periquitãomaracanã (Aratinga leucophtalmus) e Maitaca verde (Pionus maximilianus) da família Psittacidae [ordem Psittaciformes]; Tucano toco (Ramphastos 
toco) da família Ramphastidae [ordem Piciformes]; Coruja buraqueira (Athene cunicularia) da família Strigidae e Suindara (Tyto alba) da família Tytonidae [ordem Strigiformes]; Gavião carijó (Rupornis magnirostris) da família Accipitrinae, Falcão de coleira (Falco femoralis), Chimango (Mivalgo chimango), Gavião carrapateiro ( $M$. chimachima), Gavião carcará (Caracara plancus) da família Falconidae [Ciconiiformes]. Das espécies analisadas, somente as pertencentes à ordem Psittaciformes eram provenientes de cativeiro (oriundos de tráfico animal), as demais eram de vida livre.

Das 117 aves avaliadas, 20 (17.1\%) indivíduos apresentaram soroconversão positiva para algum dos agentes pesquisados. Desses, 65\% pertenciam à espécie $A$. aestiva (13/20), 20\% à $T$. alba (4/20), $10 \%$ à $M$. chimachima $(2 / 20)$ e $5 \%$ à B. magnirostris (1/20), sendo detectados anticorpos para T. gondii em 60\% (12/20), M. gallisepticum em $40 \%$ (8/20) e Salmonella spp. em $10 \%(2 / 20)$. Não foram detectados anticorpos para APMV 1 e M. synoviae neste estudo (Tabela 1).

Tabela 1. Relação das espécies aviárias analisadas durante o estudo

\begin{tabular}{|c|c|c|c|c|c|c|c|}
\hline Especies & Aves examinadas & Aves positivas & DNC & TG & $\mathrm{SP}$ & MG & $\mathrm{MS}$ \\
\hline \multicolumn{8}{|l|}{ CICONIIFORMES } \\
\hline \multicolumn{8}{|l|}{ Accipitridae } \\
\hline Rupornis magnirostris & 1 & 1 & - & 1 & - & - & - \\
\hline \multicolumn{8}{|l|}{ Falconidae } \\
\hline Falco femuralis & 2 & 0 & - & - & - & - & - \\
\hline Mivalgo chimachima & 3 & 2 & 0 & 2 & 0 & 0 & 0 \\
\hline Mivalgo chimango & 1 & 0 & - & - & - & - & - \\
\hline Caracara plancus & 2 & 0 & - & - & - & - & - \\
\hline \multicolumn{8}{|l|}{ PSITTACIFORMES } \\
\hline \multicolumn{8}{|l|}{ Psittacidae } \\
\hline Amazona aestiva & 71 & 13 & 0 & 7 & 2 & 4 & 0 \\
\hline Amazona amazonica & 1 & 0 & - & - & - & - & - \\
\hline Ara ararauna & 3 & 0 & - & - & - & - & - \\
\hline Aratinga leucophtalmus & 17 & 0 & - & - & - & - & - \\
\hline Pionus maximiliani & 1 & 0 & - & - & - & - & - \\
\hline \multicolumn{8}{|l|}{ PICIFORMES } \\
\hline \multicolumn{8}{|l|}{ Ramphastidae } \\
\hline Ramphastos toco & 10 & 0 & - & - & - & - & - \\
\hline \multicolumn{8}{|l|}{ STRIGIFORMES } \\
\hline \multicolumn{8}{|l|}{ Strigidae } \\
\hline Athene cunicularia & 1 & 0 & - & - & - & - & - \\
\hline \multicolumn{8}{|l|}{ Tytonidae } \\
\hline Tyto alba & 4 & 4 & 0 & 2 & 0 & 4 & 0 \\
\hline TOTAL & 117 & 20 & 0 & 12 & 2 & 8 & 0 \\
\hline
\end{tabular}

N- número de aves avaliadas; DNC- vírus da Doença de Newcastle PMV1; TG- Toxoplasma gondii; SP- Salmonella spp.; MGMycoplasma gallisepticum; MS- M. synoviae.

A espécie A. aestiva foi soropositiva para os três agentes avaliados, prevalecendo um maior número de indivíduos com sorologia para $T$. gondii (7/12). Dois exemplares de T. alba foram soropositivos para dois agentes distintos (T. gondii e $M$. gallisepticum). As contra-provas realizadas nas aves positivas no SAR para $M$. gallisepticum também foram positivas no teste de IH.

\section{DISCUSSÃO}

A região noroeste do estado de São Paulo, Brasil, é grande produtora de produtos avícolas, sendo que a fauna silvestre pode contribuir na manutenção de doenças para aves de produção. A monitoria da sanidade de aves silvestres pode 
contribuir na avaliação da presença de enfermidades de interesse econômico, pois as aves silvestres se contaminam devido à aproximação com aves industriais doentes (através do contato direto com os agentes etiológicos) ou, no caso das aves carnívoras, pelo fato de se alimentarem de presas previamente contaminadas (por um contato indireto), servindo como aves "sentinelas" com grande potencial para uma leitura epidemiológica das doenças estudadas.

As aves avaliadas eram mantidas em viveiros coletivos amplos ( $2 \mathrm{~m}^{2}$ por ave), sendo separadas por espécie e com concentração máxima de 12 indivíduos por viveiro. Das 20 aves positivas, os dois exemplares de A. aestiva positivos para Salmonella spp., como também os quatro exemplares de T. alba, compartilhavam o mesmo viveiro. Os demais estavam alojados em viveiros diferentes.

Após a constatação de resposta imunológica, refizeram-se três vezes (com intervalo de 15 dias) todos os exames das aves positivas para Salmonella spp. e $M$. gallisepticum e também das aves contactantes (que compartilham o mesmo viveiro), tendo-se observado a manutenção da positividade entre as aves já registradas anteriormente e a ausência de soroconversão das aves contactantes, caracterizando assim a não circulação dos agentes entre as aves durante o estudo. Antes, durante e após os exames, todas as aves positivas apresentavam ausência de sintomatologias compatíveis com quadros de salmonelose, toxoplasmose e micoplasmose, como também não houve histórico de óbitos por essas doenças.

A não observação de anticorpos para parainfluenza APMV 1 está de acordo com o observado na literatura, em que não há relatos da doença de Newcastle no Brasil com envolvimento de APMV 1 desde o ano de 2001 em aves comerciais (FRANZO, 2007). A ausência de reação solorógica para $M$. synoviae também confere com a literatura, pois esta espécie de Mycoplasma acomete especificamente aves da ordem Galliformes, não havendo registro de infecção natural em outras ordens aviárias.

O uso da técnica de SAR com antígeno para $S$. Pullorum é comumente empregado em aves de produção para pré-triagem de sorotipos de Salmonella enterica pertencentes ao sorogrupo D, pois há uma soroconversão cruzada com os demais sorotipos pertencentes a esse sorogrupo (BERCHIERI JÚNIOR \& OLIVEIRA, 2007), que abriga os sorotipos de maior interesse avícola.

Em Psittaciformes, já foi descrita a ocorrência dos sorotipos Pullorum (EHRSAM, 1985) e Enteritidis (OROSZ et al., 1992; MARIETTOGONÇALVES et al., 2010) pertencentes ao sorotipo D. Os sorotipos pertencentes a outros sorogrupos descritos em Psittaciformes são o Typhimurium (WE JÚNIOR \& HATKIN, 1978; SAWA et al., 1981; MENÃO et al., 2000), Houtenae (WERNERY et al., 1998), Arizonae (ORÓS et al., 1998), Braenderup (ALLGAYER et al., 2009) e Rissen (JANG et al., 2008). No presente estudo, não houve detecção de Salmonella pertencente ao sorogrupo D em aves de rapina, mas na literatura há relatos de isolamento de $S$. enteritidis (POVEDA et al., 1994), além de $S$. typhimurium e $S$. montevideo (ZIEDLER et at., 1995; SMITH et al., 2002), pertencentes aos outros sorogrupos.

M. gallisepticum é um patógeno aviário que pode ser encontrado tanto em aves domésticas como selvagens (LEVISOHN \& KLEVEN, 2000). A presença de antígenos contra $M$. gallisepticum em $A$. aestiva e T. alba pode ter ocorrido devido ao contato desses exemplares com outras espécies aviárias. No ambiente antrópico, o contato de aves silvestres com aves sinantrópicas portadoras clássicas de $M$. Gallisepticum, como o pombo doméstico e o pardal (HARTUP et al., 2001; KLEVEN \& FLETCHER, 1983), é frequente, muitas vezes inevitável. A utilização de SAR como forma de diagnóstico sorológico para Mycoplasma é insuficiente por não ser um teste muito específico (MARIETTOGONÇALVES et al., 2008), sendo recomendável sua utilização apenas para triagem. O teste de inibição da hemaglutinação, como o ensaio imunoenzimático de absorção em fase sólida (enzyme-linked immunosorbent assay - ELISA), é um método sorológico confirmatório recomendado, embora a confirmação definitiva implique o isolamento do agente.

Psittaciformes são altamente susceptíveis a infecção por $M$. gallisepticum, estando o agente comumente envolvido em casos de óbitos por infecções respiratórias em exemplares cativos (ADLER, 1857; BOZEMAN et al., 1984; GOMES et al., 2010). Outra espécie importante para avicultura reportada em psittacídeos é o $M$. iowa (BOZEMAN et al., 1984). A ocorrência de M. gallisepticum em aves de rapina foi descrita por ZIEDLER et al. (1995) e MORISHITA et al. (1997). A ocorrência de M. meleagridis também já foi observada em rapinantes (LIERZ et al., 2000), em que são descritos o M. falconis, M. gypis e M. buteonis como espécies específicas (POELMA \& ZWART, 1972), não havendo até o momento estudos relatando a existência de reação sorológica cruzada dessas espécies com M. gallisepticum (falso positivo).

A presença de anticorpos para $T$. gondii foi encontrada em quatro espécies, sendo observada a titulação de 1:16 nos exemplares de A. aestiva, $T$. alba, $R$. magnirostris e de 1:64 nos dois exemplares de $M$. chimachima. A ocorrência de toxoplasmose 
em Psittaciformes é incomum, sendo relatada em espécies australo-asiáticas (POELMA \& ZWART, 1972; HARTLEY \& DUBEY, 1991; HOERT et al., 1991), em diagnóstico post-mortem, não havendo registro de titulação sorológica na literatura.

Aves de rapina são consideradas refratárias ao T. gondii (DUBEY, 2002), porém é descrita a ocorrência de toxoplasmose em Strix varia (MIKAELIAN et al., 1997) e Haliaeetus leucocephalus (ZIEDLER et al., 1995). Em um estudo realizado por LINDSAY et al. (1993), exemplares do gênero Buteo apresentaram titulação de 1:80, não havendo presença de sinais clínicos. Em outro estudo, KIRKPATRICK et al. (1990) encontraram titulação de 1:50 em T. alba. AUBERT et al. (2008) também relataram a titulação de 1:25 em exemplares de Buteo e T. alba. Logo, os títulos de 1:16 encontrados em $R$. magnirostris e $T$. alba foram considerados baixos. Não foi encontrada titulação ou notificação de $T$. gondii em $M$. chimachima e $R$. magnirostris na literatura, sendo este o primeiro relato de anticorpos contra $T$. gondii nessas espécies.

Dois exemplares de T. Alba apresentaram soroconversão para $T$. gondii e $M$. gallisepticum. Ambos os agentes não apresentam qualquer tipo de correlação biológica, não sendo encontrada na literatura atual a descrição de casos de toxoplasmose associada à micoplasmose, atribuindo-se o fato observado como ocasional.

Atribuiu-se o fato de a espécie A. aestiva apresentar o maior número de indivíduos com anticorpos para alguns dos agentes neste estudo pela quantidade de exemplares estudos. Isso se dá por ser uma espécie comumente encontrada no comércio ilegal de animais no Brasil devido à grande procura como um animal pet alternativo.

Conforme os resultados observados, compreendemos que aves silvestres podem ser utilizadas como sentinela para estudos epidemiológicos que visam à monitoria da circulação ambiental de agentes biológicos de interesse avícola como também para a saúde pública, sendo que o emprego da monitoria sorológica em centros de recuperação animal é uma ferramenta útil para prevenir a disseminação de diferentes agentes patogênicos no meio ambiente por aves reintroduzidas.

\section{CONCLUSÃO}

A análise sorológica das aves estudadas demonstrou que há a circulação de Salmonella spp., $M$. gallisepticum e $T$. gondii em aves provenientes de tráfico e a circulação silvestre de $M$. gallisepticum e T. gondii em aves de rapina da região noroeste do
Estado de São Paulo, notificamos também pela primeira vez a ocorrência de sorologia positiva para T. gondii em Mivalgo chimango e Rupornis magnirostris.

\section{REFERÊNCIAS}

ADLER, H.E. Isolation of a pleuropneumonia-like organism from the air sac a parakeet. Journal of the American Veterinary Medical Association, v.130, p.408-409, 1957.

ALLGAYER, M.C.; OLIVEIRA, S.J.; MOTTIN, V.D.; LOIKO, M.R.; ABILLEIRA, F.; GUEDES, N.M.R.; PASSOS, D.T.; WEIMER, T.A. Isolamento de Salmonella Braenderup em Arara-azul (Anodorhynchus hyacinthinus). Ciência Rural, v.39, p.2542-2545, 2009.

AUBERT, D., TERRIER, M. E., DUMÈTRE, A., BARRAT, J., VILLENA, I. Prevalence of Toxoplasma gondii in raptors from France. Journal of Wildlife Diseases, v.44, p.172-173, 2008.

BOZEMAN, L.H.; KLEVEN, S.H.; DAVIS, R.B. Mycoplasma Challenge Studies in Budgerigars (Melopsittacus undulatus) and Chickens. Avian diseases, v.28, p.426-434, 1984.

CATOLLI, G.; SUSTA, L.; TERREGINO, C.; BEOWN, C. Newcastle disease: a review of field recognition and current methods of laboratory detection. Journal of Veterinary Diagnostic Investigation, v.23, p.637-656, 2011.

DESMONTS, G., REMINGTON, J.S. Direct agglutination test for diagnosis of Toxoplasma infection: method for increasing sensitivity and specificity. Journal of Clinical Microbiology, v.11, p.562-568, 1980.

DUBEY, J.P. A review of toxoplasmosis in wild birds. Veterinary Parasitology, v.106, p.121-153, 2002.

EHRSAM, H. Acute pullorum disease in hawk-headed parrots (Deroptyus accipitrinus fuscifrons). Schweizer Archiv für Tierheilkunde, v.127, p.397-400, 1985.

ELMORE, S.A.; JONES, J.L.; CONRAD, P.A.; PATTON, S.; LINDSAY, S.; DUBEY, J.P. Toxoplasma gondii: epidemiology, feline clinical aspects, and prevention. Trends in Parasitology, v. 26, p.190-196, 2010.

FRANZO, V.S. Ocorrência da doença de Newcastle no Brasil e no mundo. Revista de Ciências Veterinárias, v. 5, n.5, p.81-85, 2007.

GASKIN, J.M.; JACOBSON, E.R. A Mycoplasma associated epornitic in Severe macaws (Ara severa severa). Proceedings of the American association of Zoo Veterinarians annual meeting, p.59-61, 1979.

GOMES, A.M.; COSTA, L.L.; VILELA, D.A.R.; MARQUES, M.V.R.; CARVALHAES, A.G.; MARIN, S.Y.; COSTA, M.P.; HORTA, R.S.; RESENDE, J.S.; MARTINS, N.R.S. Detection of Mycoplasma 
gallisepticum in dead captive psittacines in Belo Horizonte, Brazil. Revista Brasileira de Ciência Avícola, v.12, p.75-78, 2010 .

HARTLEY, W.J., DUBEY, J.P. Fatal toxopalsmosis in some native Australian birds. Journal of Veterinary Diagnostic Investigation, v.3 p.167-169, 1991.

HARTUP, B.K.; DHONDT, A.A.; SYDENSTRICKER, K.V.; HOCHACHKA, W.M.; KOLLIAS, G.V. Host range and dynamics of mycoplasmas conjunctivitis among birds in North America. Journal of Wildlife Diseases, v.37, p.72-81, 2001.

HOWERT, E.W.; RICH, G.; DUBEY, J.P. Fatal toxoplasmosis in a red lory (Eos bornea). Avian Diseases, v.35, p.642-646, 1991.

JANG, Y.H.; LEE, S.J.; LIM, J.G.; LEE, H.S.; KIM, T.J.; PARK, J.H.; CHUMG, T.H.; CHOE, N.H. The rate of Salmonella spp. infection in zoo animals at Seoul Grand Park. Korean Journal of Veterinary Sciences, v.9, p.177-181, 2008.

KIRKPATRICK, C.E.; COLVIN, B.A.; DUBEY, J.P. Toxoplasma gondii antibodies in Common barn-owls (Tyto alba) and Pigeons (Columba livia) in New Jersey. Veterinary Parasitology, v.36, p.177-180, 1990.

KLEVEN, S.H.; FLETCHER, W.O. Laboratory infection of House sparrows (Passer domesticus) with Mycoplasma gallisepticum and Mycoplasma synoviae. Avian Diseases, v.27, p.308-311, 1983.

LEVISOHN, S., KLEVEN, S. H. Avian mycoplasmosis (Mycoplasma gallisepticum). Revue Scientifique et Technique, v.19, p.425-442, 2000.

LIERZ, M.; SCHMIDT, R.; BRUNNBERG, L.; RUNGE, M. Isolation of Mycoplasma meleagridis from freeranging birds of prey in Germany. Journal of Medicine Veterinary - Series B, v.47, p.63-67, 2000.

LINDSAY, D.S.; SMITH, P.C.; HOERR, F.J.; BLAGBURN, B.L. Prevalence of encysted Toxoplasma gondii in raptors from Alabama. Journal of Parasitology, v.79, p.870-873, 1993.

LOMNICZI, B.; WEHMANN, E.; HERCZEG, J.; BALLAGI-PORDANY, A.; KALETA, E.F.; WERNER, O.; MEULEMANS, G.; JORGENSESN, P.H.; MANTE, A.P., GIELKENS, A.L.; CAPUA, I.; DAMOSER, J. Newcastle disease outbreaks in recent years in western Europe were caused by an old (VI) and a novel genotype (VII). Archives of Virology, v.143, p.49-64, 1998.

LUTFUL KABIR, S.M. Avian Colibacillosis and Salmonellosis: A Closer Look at Epidemiology, Pathogenesis, Diagnosis, Control and Public Health Concerns. International Journal of Environmental Research and Public Health, v.7, p.89-114, 2010.

MAPA. Programa Nacional de Sanidade Avícola. Manual de Legislação - Programas Nacionais de Saúde Animal do brasil. Brasília: Ministério da Agricultura e Abastecimento 2009. pp.171-242. Disponível em: $<$ http://www3.servicos.ms.gov.br/iagro_ged/pdf/1766_GE
D.pdf>, acessado em 22/20/2013.

MARIETTO-GONÇALVES, G.A.; LIMA， E.T.; ANDREATTI-FILHO, R.L. Doenças respiratórios em aves atendidas no Laboratório de Ornitopatologia da FMVZ-UNESP/Botucatu-SP, Brasil, nos anos de 2005 a 2006. Archives of Veterinary Science, v.13, p.40-45, 2008.

MARIETTO-GONÇALVES, G.A.; ALMEIDA, S.M.; LIMA, E.T.; OKAMOTO, A.S.; PINCZWOSKI, P.; ANDREATTI-FILHO, R.L. Isolation of Salmonella enterica serovar Enteritidis in Blue-fronted amazon parrot (Amazona aestiva). Avian Diseases, v.54, p.151-155, 2010.

MENÃO, M.C.; BOTTINO, J.A.; BIASIA, I.; FERREIRA, C.S.A.; CALDERANO, F.F.; TAVECHIO, A.L.; FERNANDES, S.; FERREIRA, A.J.P. Infecção por Salmonella Typhimurium em Arara Azul (Anodorynchus hyacinthinus). Arquivos do Instituto Biológico, v.67, p.43-47, 2000 .

MIKAELIAN, I.; DUBEY, J.P.; MARTINEAU, D. Severe hepatitis resulting from toxoplasmosis in a Barred owl (Strix varia) from Quebec, Canada. Avian Diseases, v.41, p.738-740, 1997.

MORISHITA, T.Y.; PYONE, P.A.; BROOKS, D.L. A survey of diseases of raptorial birds. Journal of Avian Medicine and Surgery, v.11, p.77-92, 1997.

ORÓS, J.; RODRÍGUEZ, J.L.; FERNÁNDEZ, A.; HERRÁEZ, P.; ESPINOSA DE LOS MONTEROS, A.; JACOBSON, E.R. Simultaneous occurrence of Salmonella arizonae in a Sulfur crested cockatoo (Cacatua galerita galerita) and Iguanas. Avian Diseases, v.42, p.818-823, 1998.

OROSZ, S.E.; CHENGAPPA, M.M.; OYSTER, R.A.; MORRIS, P.J.; TROCK, S.; ALTEKRUSE, S. Salmonella enteritidis infection in two species of psittaciformes. Avian Diseases, v.36, p.766-769, 1992.

POELMA, F.G.; ZWART, P. Toxoplasmose bij kroonduiven en andere vogels in de Koninklijke Rotterdamse Diergaarde (Blijdorp). Acta Zoologica et Pathologica Antverpiensia, v.55, p.29-40, 1972.

POVEDA, J.B.J.; GIEBEL, J.; FLOSDORFF, J.; MEIER, J.; KIRCHHOFF, H. M. buteonis sp. nov., M. falconis sp. nov., and M. gypis sp. nov. Three species from birds of prey. International Journal of Systematic Bacteriology, v.44, p.94-98, 1994.

RECHE, M.P.; JIMÉNES, P.A.; ALVAREZ, F.; GARCÍA DE LOS RIOS, J.E.; ROJAS, A.M.; PEDRO, P. Incidence of Salmonella in captive and wild free-living raptorial birds in central Spain. Journal of Medicine Veterinary Series B, v.50, p.42-44, 2003.

SAWA, H.; HIRAI, K.; KINJO, T.; SHIBATA, I.; SHIMAKURA, S. Salmonella typhimurium infection in imported passerine and psittacine birds. Nippon Juigaku Zasshi, v.43, p.967-969, 1981.

SILVA, F.W.S.; ALVES, N.D.; AMÓRA, S.S.A.; 
TEIXEIRA, F.H.V.; ACCIOLY, M.P.; CARVALHO, C.G.; NÓBREGA, R.M.; FILGUEIRA, K.D.; FEIJÓ, F.M.C. Toxoplasmose: uma revisão. Ciência Animal, v. 16, p.71-77, 2006.

SMITH, W.A.; MAZET, J.A.; HIRSH, D.C. Salmonella in California wildlife species: prevalence in rehabilitation centers and characterization of isolates. Journal of Zoo and Wildlife Medicine, v.33, p.228-235, 2002.

TINDALL, B.J.; GRIMONT, P.A.D.; GARRITY, G.M.; EUZÉBY, J.P. Nomenclature and taxonomy of the genus Salmonella. International Journal of Systematic and Evolutionary Microbiology, v.55, p.521-524, 2005.

SZABO, K.A.; MENSE, M.G.; LIPSCOMB, T.P.; FELIX, K.J.; DUBEY, J.P. Fatal toxoplasmosis in a Bald eagle (Haliaeetus leucocephalus). Journal of Parasitology, v.90, p.907-908, 2004.

ZIEDLER, K.; HLINAK, A.; RAETZ, G.; WERNER, O.;
EBNERE, D. Untersuchungen zum antikorperstatus von Wild- und Zoovogeln gegen ausqewahlte Nutztierrelevante Erreger. Journal of Veterinary Medicine Series B, v.42, p.321-330, 1995.

WARD, M. P., RAMER, J. C., PROUDFOOT, J., GARNER, M. M., JUAN-SALLÉS, C.; WU, C. C. Outbreak of salmonellosis in a zoologic collection of lorikeets and lories (Trichoglossus, Lorius, and Eos spp.). Avian Diseases, v.47, p.493-498, 2003.

WE JÚNIOR, P.; HATKIN, M. Isolation of Salmonella houtenae from a cockateel. Avian Diseases, v.22, p.350353, 1978.

WERNERY, U.; WERNERY, A.; ZACHARIAH, R; KINNE, J. Salmonellosis in relation to chlamydiosis and pox and Salmonella infections in captive falcons in the United Arab Emirates. Journal of Veterinary Medicine Series B, v.45, p.577-583, 1998.

Protocolado em: 15 dez. 2011 Aceito em: 09 set. 2013. 